\title{
Opium withdrawal and some blood biochemical factors in addicts' individuals
}

\author{
Mehdi Mahmoodi $^{{ }^{*}}$, Seyed-Mostafa Hosseini-Zijoud ${ }^{2}$, Javad Hosseini ${ }^{2}$, Ahmadreza Sayyadi ${ }^{3}$, \\ Mohammadreza Hajizadeh $^{4}$, Gholamhossein Hassanshahi ${ }^{5}$, Behrooz Hashemi ${ }^{6}$ \\ ${ }^{1}$ Clinical Biochemistry, Molecular Medicine Research Center, Rafsanjan University of Medical Sciences, Rafsanjan, Iran \\ ${ }^{2}$ Clinical Biochemistry, Department of Biochemistry, Biophysics \& Genetics, Faculty of Medicine, Rafsanjan University of Medical \\ Sciences, Rafsanjan, Iran \\ ${ }^{3}$ Department of Psycho-Nursing, Faculty of Nursing, Midwifery and Paramedics, Rafsanjan University of Medical Sciences, Rafsanjan, Iran \\ ${ }^{4}$ Department of Biochemistry, Biophysics \& Genetics, Faculty of Medicine, Rafsanjan University of Medical Sciences, Rafsanjan, Iran \\ ${ }^{5}$ Molecular Medicine Research Center, Rafsanjan University of Medical Sciences, Rafsanjan, Iran \\ ${ }^{6}$ Shohada-e-Tajrish Hospital, Tehran, Iran \\ Email: ${ }^{*}$ mahmoodies@yahoo.com
}

Received 27 December 2011; revised 17 February 2012; accepted 24 February 2012

\begin{abstract}
Objective: One of the common misinterpretation beliefs in some societies (especially eastern communities) is the using of opium can reduce serum glucose and lipids. Opium is a derivative from a plant family called Papaveracea and contains almost 80 types of alkaloids. Drug addiction causes physiological dependency and its withdrawal lead to some disorders. The aim of this study was to determine the effects of opium consumption and its withdrawal on some blood biochemical factors in addicted people. Methods: We enrolled fifty-six opium addicted people according to the especial criteria to this study. Biochemical blood parameter levels such as fasting blood sugar (FBS), urea, Creatinine (Cr), Aspartate transaminase (AST), Alanine transaminase (ALT) and Alkaline phosphatase (ALP) enzymes levels were measured and urine analysis was also performed before and 3 months after withdrawal. Data were analyzed by using SPSS software version 18 and a $P<0.05$ was considered as significant. Results: Our finding showed that opium withdrawal reduces FBS and increases AST but these changes were not significant. Nevertheless opium withdrawal significantly increased blood urea level $(P<\mathbf{0 . 0 0 0 1})$. We didn't find any significant difference in Cr, ALP, AST and Urea specific gravity (SG). Conclusion: According to the results of the current study we can concluded that opium increases FBS, which is in contrast to the most previous studies and withdrawal has opposite effects.
\end{abstract}

Keywords: Opium; Addiction; Withdrawal; Fasting Blood Sugar (FBS)

"Corresponding author.

\section{INTRODUCTION}

The prevalence of opium addiction is $1 \%-2 \%$ worldwide. It seems that the prevalence of addiction in Iran is much higher than the rest of the world and a prevalence of $22 \%$ in some rural areas has been reported [1]. Unfortunately, the low educated people and even a limited number of physicians believe that opium has beneficial effects on cardiovascular diseases and especially diabetes. They considered opium as an anti-diabetic agent [2]. Opium is a derivative of one of the oldest plants that had cultivated by human [3]. This plant belongs to the Papaveraceae family with binomial name of Papaver somniferum. It contains more than 80 alkaloids, morphine and codeine are considered as the most important components among them [4]. Noscapine and papaverine are the other important components of opium [5]. Papaverine increases renal blood flow, urine output and $\mathrm{Cr}$ clearance [6]. Noscapine is widely used as an antitussive agent in many countries [7]. It has been reported that morphine increases hormones such as adrenalin, noradrenalin, corticosterone and glucagon, which in turn affect metabolism in different ways $[8,9]$. Morphine also indicated to increase release of melatonin from rat pineal glands, this metabolite rise mRNA expression of antioxidant enzymes [10]. Long-term addictionlead to physiological dependency and make their withdrawal more difficult. The withdrawal syndrome and drug-related deaths are still the most serious public health problem. The withdrawal syndrome occurs by following intensive drug dependency and then the administration of drug suddenly stopped or decreased in dosage. In withdrawal syndrome, the central nervous system, gastrointestinal tract are involved and it seems that this may also follow an autoimmunity pattern [11]. Dependency and withdrawal status 
may induce oxidative stress leading to production of free radicals [12]. A study showed that heroin (diacetylmorphine, an opioid that is derived from morphine) diminished levels of some serum trace elements such as Selenium (Se), Titanium (Ti), Magnesium (Mg), Manganese $(\mathrm{Mn})$ and Iron $(\mathrm{Fe})$ and rose levels of Phosphore $(\mathrm{P})$ and Aluminum (Al). Furthermore, withdrawal caused reduction ofserum $\mathrm{Se}, \mathrm{Ti}, \mathrm{Mg}, \mathrm{Mn}$ and Fe levels in parallel with increasing Copper $(\mathrm{Cu}), \mathrm{Ca}$ and $\mathrm{Al}$ levels [13]. These variations in serum bio elements levels (as decreases and increases of the antioxidant enzyme activities) might be considered as a response to increased oxidative stress mediator in either drug dependency or withdrawal [14]. It has also been established that exogenous antioxidants pretreatment lessen the severity of heroin withdrawal syndrome [13]. Moreover, it was demonstrated that opium decreases FBS temporarily in diabetic patients but it had no clear and long-lasting effects on blood glucose and $\mathrm{HbA1c}$, and triglyceride (TG), Low-density lipoprotein (LDL), High-density lipoprotein (HDL) and cholesterol levels [15]. It has been revealed that opium did not have a certain impact on glucose metabolism and serum lipid profile in diabetic rats [16]. Therefore, recent studies reported controversial findings in terms of the effects of opium on serum electrolytes, lipid markers and glucose metabolism [4]. In addition to the above factors, opium addiction caused liver damages, and increased the activity of AST, ALT [17]. A number of mentioned advantages for opium such as reducing FBS are simultaneous with more deleterious effects like rising $\mathrm{HbAlc}$, non-enzymatic glycation and lipid peroxidation [18]. Giving information to the public regarding these hidden and real side effects and disadvantages of opium consumption may modify some misunderstanding and misconception about this kind of drugs. Therefore, we designed the present study to examine the effects of opium consumption and its withdrawal on some blood biochemical factors and urine analysis in addicted individuals who referred to Rafsanjan University of Medical Sciences (RUMS) self-introduced center.

\section{MATERIALS AND METHODS}

In this interventional semi experimental study we enrolled 56 opium addicts among individuals who referred to RUMS self-introduced center. All of the individuals who didn't have any metabolic disorders which may intervene with biochemical factors, cardiovascular, mental, kidney and other chronic diseases were entered to the study. Subjects who had history of drug injection or alcohol drinking after withdrawal were also excluded from the study. All of participants filled out Fagerström questionaries' for nicotine dependency (The Fagerström Test for nicotine dependency is a standard instrument for assessing the intensity of this physical addiction), and only the subjects with DSM-IV qualification (the Diagnostic and Statistical Manual of Mental Disorders, Fourth Edition) have participated in the study. All subjects were completely informed of the plan and filled out an informed consent form prior to entering in the study. The weight and height of participants was measured and recorded at the beginning and 3 month after withdrawal. The BMI (Body Mass Index) in each step was also calculated. The way and the amount and the type of opium, and other drug consumption were determined, and then blood and urine samples were collected. The dependency or independency was determined before and after withdrawal by morphine test and the individuals who had started using of opium after withdrawal were excluded from the study. Blood and urine samples were collected before and 3 month after withdrawal. FBS, Urea, Cr, ALT, AST and ALP were measured by automated Autoanalyser BT-3000 instrument and urine analysis was carried out with urine strip and light microscopy method. The obtained data were analyzed using Student T-test and repeated measure was used for comparing the means. Significant level was considered as $\mathrm{P}<$ 0.05 .

\section{RESULTS AND DISSCUSION}

Results of our study displayed that there was only a significant increase in the urea level following 3 months of opium withdrawal $(P=0.0001)$. It was observed no statistically significant differences in the serum level of FBS, AST, Cr, ALP and urine specific gravity (Table 1).

Narcotic drug addiction is considered as a prominent problem of 21 st century worldwide. Iran is located on the traffic band of narcotics and, hence, major consumer of drugs, especially opium [19]. Unfortunately, there is a public believe that opium can lead to decreased serum glucose and lipid levels in diabetes so tends to use these kinds of drugs [14]. Opium and its alkaloids consumed as hypoglycemic agent in Europe [20].

In drug addiction the most involved organs are central nervous system [21], endocrine and urinary system, hematological and biochemical parameters [22,23]. Longterm consumption of opioids especially opium, alter the homeostasis and many studies have been carried out in this field [20]. In the present study opium effects on some blood biochemical factors and urine analysis were measured in opium addicts before and 3 months after withdrawal (Table 1). Current results displayed a profile in which withdrawal decreased FBS while increased Urea and AST and the statistical differences only for urea was significant $(P=0.0001)$. The biochemical factors such as ALT, ALP, Cr and urine specific gravity (SG) didn't show any significant difference (Table 1). So based on our findings and the above introductory docu- 
Table 1. Mean of blood factors and urine specific gravity (SG) before and after withdrawal (mean $\pm \mathrm{SEM}$ ), $\mathrm{N}=56$.

\begin{tabular}{cccc}
\hline Factor & Before Withdrawal & After Withdrawal & Description \\
\hline FBS $(\mathrm{mg} / \mathrm{dl})$ & $101.7 \pm 4$ & $95.3 \pm 2.5$ & Reduced-Not Sig. \\
Urea $(\mathrm{mg} / \mathrm{dl})$ & $12.5 \pm 0.5$ & $27.8 \pm 8$ & Increased-Sig (0.0001) \\
Cr (mg/dl) & $0.81 \pm 0.02$ & $0.79 \pm 0.02$ & Not Sig. \\
ALP $(\mathrm{IU} / \mathrm{L})$ & $260.2 \pm 9.7$ & $246.8 \pm 9$ & Not Sig. \\
AST $(\mathrm{IU} / \mathrm{L})$ & $23.9 \pm 1.9$ & $28.8 \pm 2.5$ & Not Sig. \\
ALT $(\mathrm{IU} / \mathrm{L})$ & $25.5 \pm 5.8$ & $26.4 \pm 1.2$ & Not Sig. \\
SG $(\mathrm{mg} / \mathrm{ml})$ & $1021 \pm 8$ & $1023 \pm 26$ & Not Sig. \\
\hline
\end{tabular}

Sig. = Significant.

ments we can suggest that opium may increase blood glucose, in contrast to some previous data that reported hypoglycemic effect of opium.

Diaz et al. evaluated blood biochemical and hematological parameters in addicts and reported. Reduced number of red cells, decreased cholesterol, albumin, retinol, alpha-Tocopherol, folic acid, Potassium (K) and $\mathrm{Se}$, but increased the levels of Sodium (Na), ALT, AST and the numbers of leukocytes in addicted people in compare to control subjects [23]. Opium is considered as leading cause of liver damages, and a significant relationship between opium consumption and grade of liver damages in the patients with hepatitis was observed. The ALT and AST levels were constantly higher in opium addicts which in a way confirms that opium consumption have significant effects on hepatitis and its complications [24].

In the current study we found that addicted diabetic males showed higher levels of $\mathrm{HbA1c}, \mathrm{Fe}, \mathrm{K}$ and uric acid and lower levels of total protein, total cholesterol, albumin, ALT and HDL compare to non-addicted diabetic patients but the changes in other factors weren't significant. Also in dependent diabetic female's total iron binding capacity (TIBC), Total protein, ALT and AST levels were lower and HbAlc level was higher than non-addicted diabetics. So we suggest that opium possibly exerts metabolic disorders in diabetic patients via increasing serum glucose and diminishing HDL level [4]. In another study that was carried out by our group again we observed that glucose, $\mathrm{Na}$ and $\mathrm{K}$ levels increased in opium received rats compared to control group [25].

In agreement with our findings Helmstädter et al. reported, no changes were found in $\mathrm{Na}, \mathrm{Ca}$, blood urea nitrogen (BUN), TG, Cr and total Protein levels among; opium addicts, heroin addicts and healthy control, but FBS, K and uric acid levels were significantly lower in opium addicts compared to healthy control. They showed that serum $\mathrm{Ca}$ concentration was decreased in heroin addicts in compare to healthy group. Both addicted groups opium and heroin showed decreased serum cho- lesterol levels [20].

Overall, in the current study some biochemical factors in opium addicts, before and after withdrawal was evaluated, some findings were consistent and some were against previous studies. According to the results of our present study, it was found that opium caused an elevation in FBS in contrast to previous studies and the opium withdrawal decreased FBS but this was not significant, thus, it can be concluded that opium is not a useful tool for hypoglycemia as is believed by many people.

In conclusion according to the obtained results of this study we suggest that opium consumption in diabetic patient isn't useful in spite of public opinion and some of the previous findings. It has also been found that opium withdrawal had no effect on lipid profiles.

\section{REFERENCES}

[1] Bashardoost, N. and Tirani, M. (2005). Prevalence of addiction in males: Isfahan 2003. ARYA Journal, 1, 106108.

[2] Frick, S., Kramell, R., Schmidt, J., et al. (2005) Comparative qualitative and quantitative determination of alkaloids in narcotic and condiment Papaver somniferum cultivars. Journal of Natural Products, 68, 666-673. doi:10.1021/np0496643

[3] Frick, S., Kramell, R. and Kutchana, T.M. (2007) Metabolic engineering with a morphine biosynthetic P450 in opium poppy surpasses breeding. Metabolic Engineering, 9, 169-176. doi:10.1016/j.ymben.2006.10.004

[4] Karam, G.., Reisi, M., Kaseb, A.A., et al. (2004) Effect's opium addiction on some serum factors in addicts with non-insulin-dependent diabetes mellitus. Addiction Biology, 9, 53-58. doi:10.1080/13556210410001674095

[5] Gutstien, H.B. and Akil, H. (2006) Opioid analgesics. In: Brunton, L.L., Lazo, J.S. and Parker, K.L., Eds., The Pharmacological Basis of Therapeutics, 11th Edition, McGrow-Hill, New York, 547-584.

[6] Zacherl, J., Thein, E., Stangl, M., et al. (2003) The influence of periarterial papaverine application on intraopera- 
tive renal function and blood flow during laparoscopic donor nephrectomyin a pig model. Surgical Endoscopy, 17, 1231-1236. doi:10.1007/s00464-002-8835-5

[7] Landen, J.W., Hau, V., Wang, M., et al. (2004) Noscapine crosses the blood brain barrier and and inhibits glioblastoma growth. Clinical Cancer Research, 10, 5187-5201. doi:10.1158/1078-0432.CCR-04-0360

[8] Lesile, J.K. and Taborsky, G.Y. (1986) Rele of pancreatic somatostatinin determing glucagon response to arginine and morphine. American Journal of physiology, 252, 750755.

[9] Ipp, E., Dobbs, R. and Unger, R.H. (1978) Morphine and beta-endorphin influence the secretion of the endocrine pancreas. Nature, 276, 190-191. doi:10.1038/276190a0

[10] Esposti, D., Esposti, G., Lissoni, P., et al. (1998) Action of morphine on melatonin release in the rat. Journal of Pineal Research, 5, 35-39. doi:10.1111/j.1600-079X.1988.tb00766.x

[11] Pinelli, A., Cighetti, G. and Trivulzio, S. (2008) Plasma malondialdehyde levels and opiate withdrawal signs observed in rats treated with morphine plus naloxone: Effects of $\alpha$-lipoic acid administration. Fundamental \& Clinical Pharmacology, 22, 439-445. doi:10.1111/j.1472-8206.2008.00612.x

[12] Singhal, P.C., Pamarthi, M., Shah, R., et al. (1994) Morphine stimulates superoxide formation by glomerular mesangial cells. Inflammation, 18, 293-299. doi:10.1007/BF01534270

[13] Cemek, M., Büyükokuroğlu, M.E., Hazman, O., et al. (2011) Antioxidant enzyme and element status in heroin addiction or heroin withdrawal in rats: Effect of melatonin and Vitamin E plus Se. Biological Trace Element Re-search, 139, 41-54. doi:10.1007/s12011-010-8634-0

[14] Cemek, M., Büyükokuroğlu, M.E., Yürümez, Y., et al. (2010) Tissue trace and major element levels in organophosphate insecticide fenthion $\left(\right.$ Lebaycid $^{\mathbb{R}}$ ) toxicity in rats: Prophylactic and therapeutic effect of exogenous melatonin. Ecotoxicology and Environmental Safety, 73, 206-212. doi:10.1016/j.ecoenv.2009.09.008

[15] Azod, L., Rashidi, M., Afkhami-Ardekani, M., et al. (2008) Effect of opium addictionon diabetes. American Journal of Drug Alcohol Abuse, 34, 383-388.

\section{doi: $10.1080 / 00952990802122580$}

[16] Sadeghian, S., Boroumand, M., Sotoudeh-Anvari, M., et al. (2009) Effect of opium on glucose metabolism and lipid profiles in rats with streptozotocin-induced diabetes. Endokrynol Pol, 60, 258-262.

[17] Kharchenko, N.K., Synyts'kyi, V.N. and Kovtun, T.V. (2001) Comparative analysis of the effects of alcoholism and opium addiction on liver function. Fiziol $\mathrm{Zh}, 47,81$ 86.

[18] Hussein, O.A., Gefen, Y., Zidan, J.M., et al. (2007) LDL oxidation is associated with increased blood hemoglobin A1c levels in diabetic patients. Clinica Chimica Acta, 377, 114-118. doi:10.1016/i.cca.2006.09.002

[19] Divsalar, K., Haghpanah, T., Afarinesh, M., et al. (2010) Opium and heroin alter biochemical parameters of human's serum. American Journal of Drug Alcohol Abuse, 36, 135-139.

[20] Helmstädter, A., (2007) Antidiabetic drugs used in Europe prior to the discovery of insulin. Pharmazie, 62, 717-720.

[21] Jerome, H.J. (1980) Drug addiction and drug abuse. In: Goodman, L.S. and Gilman, A., Eds., The Pharmacological Basis of Therapeutics, 6th Edition, Macmillan Publishing Co., Inc., New York, 535-584.

[22] Shi, J., Li, S.X., Zhang, X.L., et al. (2009) Time-dependent neuroendocrine alterations and drug craving during the first month of abstinence in heroin addicts. American Journal of Drug Alcohol Abuse, 35, 267- 272. doi:10.1080/00952990902933878

[23] Díaz-Flores, E.J.F., Díaz-Flores, E.F., et al. (2004) Application of linear discriminant analysis to the biochemical and haematological differentiation of opiate addicts from healthy subjects: A case-control study. European Journal of Clinical Nutrition, 58, 449-455.

[24] Shavakhy, A., Sadeghi, A. and Minakary, M. (2010) Opium consumption and risk of liver fibrosis in chronic hepatitis B and C. Journal of Isfahan Medical School, 28, 451-458.

[25] Karam, G., Rashidinejad, H., Aghaee, M., et al. (2008) Opium can differently alter blood glucose, sodium and potassium in male and female rats. Pakistan Journal of Pharmaceutical Sciences, 21, 180-184. 